\title{
Avaliação de resto-ingesta em uma unidade de alimentação e nutrição de um
}

\section{restaurante comunitário}

\author{
Evaluation of rest-of-ingestion in food and nutrition unit from a community restaurant \\ Evaluación de resto-ingesta en unidad de alimentación y nutrición de un restaurante comunitário
}

Recebido: 09/02/2022 | Revisado: 16/02/2022 | Aceito: 25/02/2022 | Publicado: 05/03/2022

Flávia da Silva Taques Vieira

ORCID: https://orcid.org/0000-0001-8837-9059 Instituto Federal de Educação Ciência e Tecnologia de Mato Grosso, Brasil

E-mail: taquesflavia@gmail.com

Eudes Thiago Pereira Ávila

ORCID: https://orcid.org/0000-0002-2929-567X Faculdade Fasipe, Brasil

E-mail: eudes.avila@gmail.com

Anna Luiza Silva Gonzaga

ORCID: https://orcid.org/0000-0002-6446-9057 Universidade do Estado de Minas Gerais, Brasil E-mail: aluizasilvag@gmail.com

João Henrique Coelho

ORCID: https://orcid.org/0000-0002-2045-8276 Universidade do Estado de Minas Gerais, Brasil

E-mail: joao-henrique-1993@ hotmail.com

Maria Olímpia Ribeiro do Vale Almada

ORCID: https://orcid.org/0000-0002-6062-3387

Universidade do Estado de Minas Gerais, Brasil E-mail: maria.olimpia@unemat.br

\begin{abstract}
Resumo
O desperdício de alimentos é um problema mundial, haja visto que um terço dos alimentos destinados ao consumo são desperdiçados, trazendo consigo importantes impactos sociais e econômicos, $\mathrm{O}$ presente estudo teve como objetivo avaliar o índice de resto-ingesta, sobras limpas e sobra descartada do almoço da Unidade de Alimentação e Nutrição do restaurante comunitário da Fundação de Ensino Superior de Passos da Universidade do Estado de Minas Gerais. Metodologia: estudo observacional com abordagem quantitativa por 30 dias. O peso das preparações produzidas, da sobra limpa e sobra descartada e de resto ingesta, foram levantados com o uso de uma balança digital descontando-se os pesos dos recipientes. Resultados: resto ingesta 3,1\%; sobras limpas 32,52\%; ambiente, sabor (ótimo ou bom) 98,6\%; 95,6\% respectivamente; aparência dos alimentos ótimo 65,0\% e bom $27,35 \%$; temperatura dos alimentos (ótimo ou bom) 82,5\% mesmo com o valor satisfatório nessa variável houve um percentual regular e ruim de 15,5\%; variedade dos alimentos (ótimo ou bom) 89,5\%. Conclusão: o resto ingesta dos comensais apresenta valores satisfatórios, a unidade apresenta um elevado índice de desperdício, principalmente de sobras limpas, dessa forma, faz-se necessário investir em capacitação dos colaboradores, adequação do cardápio, quantidades de produção, entre outras medidas que visem reduzir o desperdício de alimentos.
\end{abstract}

Palavras-chave: Resto-ingesta; Desperdício; Unidade de alimentação e nutrição; Sobra.

\begin{abstract}
Food waste is a worldwide problem, with a third of food destined for consumption is wasted, bringing with it important social and economic impacts. The aim of this study was to evaluate the leftovers and rest-of-ingestion intake index of the lunch at the Food and Nutrition Unit of the community restaurant of the Passos Higher Education Foundation of the State University of Minas Gerais. Methodology: observational study with a quantitative approach for 30 days. The weight of the preparations produced, the clean leftovers and discarded leftovers and the : restingestion, were measured using a digital scale, deducting the weights of the recipients. Results: rest-ingestion 3.1\%; clean leftovers $32.52 \%$; environment, taste (great or good) $98.6 \%$; $95.6 \%$ respectively; food appearance great $65.0 \%$ and good $27.35 \%$; food temperature (excellent or good) $82.5 \%$, even with the satisfactory value for this variable, there was a regular and bad percentage of $15.5 \%$; variety of food (great or good) $89.5 \%$. Conclusion: the rest-ingestion has satisfactory values, the unit has a high rate of waste, especially clean leftovers, thus, it is necessary to invest in employee training, adaptation of the menu, production quantities, among other measures that aimed at reducing food waste.
\end{abstract}

Keywords: Rest-ingestion; Waste; Unit food and nutrition; Leftover. 


\begin{abstract}
Resumen
El desperdicio de alimentos es un problema a nivel mundial, ya que se desperdicia un tercio de los alimentos destinados al consumo, trayendo consigo importantes impactos sociales y económicos Almuerzo en la Unidad de Alimentación y Nutrición del restaurante comunitario de la Fundación de Educación Superior Passos de la Universidad del Estado de Minas Gerais. Metodología: estudio observacional con enfoque cuantitativo durante 30 días. El peso de los preparados elaborados, de los sobrantes limpios y desechados y del resto ingeridos, se midió mediante una balanza digital, descontando los pesos de los recipientes. Resultados: consumo de reposo 3,1\%; sobras limpias 32,52\%; ambiente, sabor (excelente o bueno) 98,6\%; 95,6\% respectivamente; aspecto de los alimentos excelentes $65,0 \%$ y bueno $27,35 \%$; temperatura de los alimentos (excelente o buena) 82,5\%, aún con el valor satisfactorio para esta variable, hubo un porcentaje regular y malo de $15,5 \%$; variedad de comida (excelente o buena) $89.5 \%$. Conclusión: la ingesta del resto de los comensales tiene valores satisfactorios, la unidad tiene un alto índice de desperdicio, sobre todos sobras limpias, por lo que es necesario invertir en capacitación de los empleados, adecuación del menú, cantidades de producción, entre otras medidas que apuntan en la reducción del desperdicio de alimentos.
\end{abstract}

Palabras clave: Resto de la ìngestión; Despercicio; Unidad de alimentación y nutrición; Sobras.

\title{
1. Introdução
}

O desperdício de alimentos é um problema de grande relevância em nível mundial, que gera impactos sociais, econômicos e ambientais. Dados da Organização das Nações Unidas para Agricultura e Alimentação (Food et al., 2015), apontam que anualmente cerca de um terço dos alimentos produzidos em todo mundo não é consumido pela população, sendo perdido em todos os setores da cadeia alimentar, desde a produção primária ao consumidor.

Esse desperdício coloca o Brasil no ranking dos 10 países que mais perdem alimentos no mundo, com cerca de $35 \%$ da produção desperdiçada anualmente (Food et al., 2015), equivalente a 39 mil toneladas por dia (Torrent et al., 2018). Em nível mundial, aproximadamente 350 milhões de toneladas de alimentos são desprezados durante a etapa de consumo (WFP, 2013), sendo que no setor de refeições coletivas e cozinhas as perdas variam de 15 a $20 \%$ (Moura et al., 2012; Silva et al., 2010).

Unidades de Alimentação e Nutrição (UANs) são unidades da área de alimentação coletiva, que têm como objetivos preparar e fornecer refeições equilibradas nutricionalmente, seguindo padrões higiênico sanitários estabelecidos pelas normas sanitárias vigentes. É comumente situada em empresas, universidades, hospitais, orfanatos, dentre outras instituições, desempenhando atividades relacionadas à alimentação e à nutrição, contribuem para manter ou recuperar a saúde de coletividades, e auxiliar no desenvolvimento de hábitos alimentares saudáveis (Borges et al., 2019).

Nesse contexto é importante destacar o papel no profissional nutricionista dentro das UANs, que tem como atribuições previstas, pelo Conselho Federal de Nutricionistas (CFN) realizar assistência e educação nutricional à coletividade ou indivíduos sadios ou enfermos em instituições públicas e privadas, além de implantar e supervisionar o controle periódico das sobras, resto ingesta e análise de desperdícios, promovendo consciência social, ecológica e ambiental dos comensais (BRASIL, 2018).

O desperdício de alimentos tem grande relevância nas UANs, pois envolve além de questões éticas, questões econômicas com reflexos políticos e sociais (Galian et al., 2016). Este pode ter origem nas sobras e nos restos. As sobras são todo o excedente de alimentos industrializados, in natura, pré-preparados ou prontos para o consumo e que não foram utilizados no dia de sua preparação. Elas podem ser classificadas em limpas e sujas ou resto. As sobras limpas são os alimentos prontos que não foram distribuídos, ficando no balcão térmico ou refrigerados sob monitoramento. As sobras sujas ou resto são os alimentos prontos que foram servidos e que não deverão ser reaproveitados, ou aqueles que ficaram em espera sem monitoramento de tempo e temperatura (Silva et al., 2010; Zandonadi et al., 2012).

Existem múltiplos fatores que influenciam o desperdício de alimentos em serviços de alimentação, o alto percentual de sobras deriva de fatores concernentes à produção de refeições, entre eles, planejamento inadequado; deficiência ou falta de treinamento de funcionários para o porcionamento; erro no dimensionamento de utensílios; ausência de pesquisa dos hábitos 
alimentares dos comensais. Enquanto, percentual elevado de restos nas bandejas pode significar a não aceitação do cardápio pelo comensal devido ao tipo de preparação ou aos aspectos sensoriais, preferencias alimentares, falta de apetite e ausência de conscientização do usuário quanto ao desperdício (Moura et al., 2012; Silva et al., 2010). Outros fatores de origem econômicas, políticas, culturais e tecnológicas, que abrangem as principais etapas da cadeia de movimentação como produção, transporte, comercialização, sistema de embalagem e armazenamento também podem acarretar essas perdas (Araújo et al., 2019; Paiva et al., 2015; Silva et al., 2010).

Consideram que desperdício é sinônimo de falta de qualidade em uma UAN, já que a quantidade de sobras e restos está ligada à adequação e aceitação do produto oferecido ao cliente, e esta inadequação pode ser evitada por meio de um planejamento adequado, sem excessos de produção e consequentemente sobras (Paiva et al., 2015).

As UANs podem avaliar a qualidade e quantidade dos alimentos servidos por meio do controle e gerenciamento do resto-ingesta (RI). O RI é a relação entre o resto devolvido nas bandejas pelo comensal e a quantidade de alimentos e preparações alimentares oferecidas, expressa em percentual. Admite-se como percentuais aceitáveis de RI taxas inferiores a $10 \%$ para pessoas sadias e $20 \%$ para pessoas enfermas, valores acima destes percentuais sugerem que os cardápios não estão adequados (Vaz, 2006). O controle de RI visa avaliar a adequação das quantidades preparadas em relação ao porcionamento na distribuição e a aceitação do cardápio por meio dos alimentos não consumido pelos clientes (De Brito et al., 2016; Silva et al., 2010)

A análise de índices como sobras limpas e restos é de grande relevância diante dos custos aumentados pelas perdas evitáveis, possibilitando o aproveitamento de recursos de forma consciente no gerenciamento de um serviço de alimentação ao investigar causas do desperdício e atuar de forma estratégica para corrigi-las. Frente ao exposto, este estudo justifica se por contribuir para a análise de quantidade de resíduos gerados, com a intenção de reduzi-los, propondo estratégias de intervenções para melhorar a qualidade do serviço prestado, a aceitação da alimentação e reduzir o desperdício.

O presente estudo teve como objetivo avaliar o índice de resto-ingesta, sobras limpas e sobra descartada do almoço da Unidade de Alimentação e Nutrição comunitária uma região de Minas Gerais.

\section{Metodologia}

A presente pesquisa consiste em um estudo observacional com abordagem quantitativa, foi realizada no restaurante comunitário da Fundação de Ensino Superior de Passos da Universidade do Estado de Minas Gerais (FESP/UEMG), que tem como objetivo o fornecimento de refeições com qualidade, balanceada e com um custo acessível aos alunos, servidores e comunidade em geral. Além de absorver os alunos do curso de nutrição para estágios curricular, extracurricular e projetos de pesquisa e extensão.

Atualmente são servidas aproximadamente 200 refeições no almoço. O restaurante conta com 9 funcionários: (1) nutricionista, (3) auxiliares de cozinha, (3) cozinheiros, (1) estoquista/caixa e (1) serviço gerais.

A estrutura do restaurante é considerada modelo em comparativo com as normas técnicas da legislação RDC n 216 contendo áreas especificas para distribuição, pré-preparo, armazenamento e cocção dos alimentos (Brasil, 2004).

O cardápio é composto por arroz, feijão, dois tipos de proteína a escolha do cliente, uma guarnição, um legume cozido e oito tipos de salada que são servidas pelo próprio cliente, em pratos de porcelana, juntamente com suco e sobremesa.

O projeto de pesquisa bem como os Termos de Consentimento Livre e Esclarecido foram submetidos e aprovados pelo Comitê de Ética em Pesquisa (CAAE: 98422818.0.0000.5112) UEMG- Universidade do estado de Minas Gerais.

A coleta dos dados foi realizada nos dias uteis do período de 01 a 31 julho de 2018. Foram avaliados as sobras e o resto-ingesta do almoço de todos os comensais (colaboradores e clientes) da Unidade de Alimentação e Nutrição Comunitária.

Os dados referentes ao peso das preparações produzidas, da sobra limpa e sobra descartada e de resto ingesta, foram 
levantados com o uso de uma balança digital, capacidade $15 \mathrm{~kg}$, divisão de $5 \mathrm{~g}$, e de uma balança manual, capacidade $200 \mathrm{~kg}$, descontando-se os pesos dos recipientes. Foram consideradas as sobras limpas parte das preparações produzidas que foram acondicionadas em recipientes adequados e armazenadas em temperatura adequada para utilização posterior. E sobra descartada as preparações não servidas que ficaram no balcão de distribuição até a finalização das refeições.

O peso do resto foi obtido por meio da pesagem do cesto de lixo, situado na área de devolução de bandejas, que continham os alimentos desprezados, descontando-se o peso do cesto. Foram excluídos partes de alimentos não comestíveis, como cascas e ossos. Os colaboradores foram orientados quanto ao uso de uma lixeira própria para o descarte do resto, não sendo permitido o descarte de mais nada além das sobras das bandejas e pratos dos comensais.

Após a quantificação do desperdício alimentar, realizou-se uma pesquisa de satisfação com os comensais para avaliar o ambiente e as preparações servidas, conforme a Figura 1. Os comensais classificaram o ambiente, sabor, aparência, temperatura e variedades das preparações servidas segundo questões objetivas, e uma questão subjetiva para justificar possíveis restos alimentares deixado no prato. As fichas foram depositadas em uma caixa de madeira fechada para o sigilo das informações, a caixa era trocada de acordo com os horários das refeições, que intercalavam entre 11:00 - 12:00; 12:00 - 13:00 e 13:00 as 13:30. Os resultados foram avaliados por meio de planilha eletrônica do Excel 2016 e demonstrados em formato de gráfico para melhor interpretação.

Figura 1: Pesquisa de satisfação.

Obrigada por escolher o restaurante comunitário FESP.
Para aprimorar nossos serviços, por favor, deixe sua opinião:
DATA:
AMBIENTE (HIGIENE):
( ) Ótimo ( ) Bom ( ) Regular ( ) Ruim
SABOR DA REFEICÃO:
( ) Ótimo ( ) Bom ( ) Regular ( ) Ruim
( ) Ótimo ( ) Bom ( ) Regular ( ) Ruim
TEMPERATURA DA REFEIÇÃO:
( ) Ótimo ( ) Bom ( ) Regular ( ) Ruim
VARIEDADE DAS PREPARACÕES SERVIDAS:
( ) Ótimo ( ) Bom ( ) Regular ( ) Ruim
VOCÊ DEIXOU COMIDA NO PRATO? SE SIM, QUAL MOTIVO?
RESPOSTA:

Fonte: Autores.

A partir dos dados coletados, foram calculadas as variáveis de análise com base nas fórmulas citadas por Vaz

- Porção de refeição distribuída (g):

Peso da refeição distribuída $(\mathbf{K g})=$ total produzido - sobras prontas após servir as refeições

- Consumo per capita por refeição:

Consumo per capita por refeição $(\mathbf{K g})=$ peso da refeição distribuída / número de refeições 
- Percentual de sobra:

\% de sobras = sobras prontas após servir as refeições x 100 / peso da refeição distribuída

- Quantidade média de sobra por cliente:

Peso da sobra por cliente $(\mathbf{K g})$ = peso das sobras / número de refeições servidas

- Índice do resto de ingestão:

$\%$ de resto-ingesta $=$ peso do resto $\times 100 /$ peso da refeição distribuída

- Resto de ingesta per capita:

Per capita do resto ingesta $(\mathbf{K g})=$ peso do resto / número de refeições servidas

Para calcular o desperdício foram consideradas o número de pessoas que poderiam ser alimentadas com a sobra e o resto acumulados durante o período de coleta de dados, por meio das equações:

Pessoas alimentadas com a sobra acumulada $=$ sobra acumulada $/$ consumo per capita por refeição

Pessoas alimentadas com o resto acumulado $=$ resto acumulado $/$ consumo per capita por refeição

Após o cálculo das variáveis acima, pôde-se fazer uma avaliação do desperdício na UAN estudada.

\section{Resultados e Discussão}

Durante o período de coleta de dados, foram servidas 2.915 refeições. Essas foram destinadas aos comensais, colaboradores e funcionários do restaurante, distribuídas conforme exposto na Tabela 1.

Tabela 1 - Distribuição das refeições servidas na UAN quanto ao número de comensais, quantidade distribuída, e consumo per capita Passos-MG, 2018.

\begin{tabular}{cccc}
\hline Dias Avaliados & Número de Comensais & Quantidade Distribuída & Consumo per capita por \\
& & $(\mathrm{kg})$ & refeição $(\mathrm{g})$ \\
22 & $133,7 \pm 23,3$ & $73,6 \pm 15,5$ & $551,2 \pm 108,8$ \\
\hline
\end{tabular}

Resultados apresentados em média e desvio padrão. Fonte: Autores.

Os dados apresentados na Tabela 2 mostram que o desperdício em percentual de resto ingesta foi satisfatório, considerando a valor máximo aceitável de 10\% para pessoas sadias (Vaz, 2006). Segundo Parisoto et al.,2013 o índice de resto ingesta (\%) pode ser classificado como ótimo (0 a 3\%), bom (3,1 a 7,5\%), ruim (7,6 a 10\%) e inaceitável (acima de 10\%). Diante do exposto, o percentual de resto ingesta encontrado pode ser classificado como bom, tendo um índice médio de 3,1\%, o equivalente a $21,9 \mathrm{~g}$ de restos de alimentos por pessoa.

Estes valores são semelhantes ao estudo realizado por De Brito et al., 2016, em que a unidade apresentou percentuais de $1,63 \%$ a $8,85 \%$, tendo uma média de 4,64\%. Segundo o autor, os bons índices contra o desperdício são decorrentes da aceitabilidade satisfatória do cardápio entre os comensais. E que estes índices podem ser atribuídos ao gerenciamento do serviço, que visa a satisfação do cliente.

Tabela 2 - Média de resto-ingesta, resto-ingesta por pessoa e número de pessoas que poderiam ser alimentadas de uma UAN Passos-MG, 2018.

\begin{tabular}{ccccc}
\hline Dias Avaliados & Número de Comensais & \% de resto-ingesta & $\begin{array}{c}\text { Per capita do resto- } \\
\text { ingesta }(\mathrm{g})\end{array}$ & PPAA \\
22 & $133,7 \pm 23,3$ & $3,1 \pm 1,42$ & $21,91 \pm 7,718$ & $5,38 \pm 1,76$ \\
\hline
\end{tabular}

Resultados apresentados em média e desvio padrão. PPAA pessoas poderiam ser alimentadas com o resto acumulado. Fonte: Autores. 
Em um estudo realizado em no instituto federal do Piauí em 2012 observaram que os índices de restos e sobras acima do limite aceitável, ocasionam custos desnecessários ao estabelecimento (Moura et al., 2012). Altos índices de resto ingesta podem ter como causa de cardápios inadequados (Vaz, 2006). Sendo necessária a adoção de medidas, como a diminuição do desperdício, planejamento adequado no processo de produção, organização de campanhas voltadas aos comensais e treinamento com os manipuladores para melhorar esses índices (Henz et al., 2017; Moura et al., 2012).

As campanhas de conscientização relacionadas ao desperdício de alimentos ajudam a controlar o resto ingesta e diminuir perdas evitáveis (Machado et al., 2012; Moura et al., 2012; Vaz, 2006). Em um estudo que realizou avaliação de resto ingesta em duas etapas, antes e após uma ação de conscientização sobre o desperdício, mostraram redução da média de resto ingesta per capita de 60,9g para 55,3 g após a intervenção (Machado et al., 2012).

A Tabela 3 apresenta em média o valor obtido da sobra limpa de alimentos no período da coleta de dados. Admite-se como aceitáveis percentuais de até $3 \%$ ou de 7 a 25 g por pessoa, sendo os valores encontrados acima do preconizado (Vaz, 2006). As sobras per capita e percentual do presente estudo também foram superiores as obtidas ao estudo de Moura em 2012, em que a sobra per capita foi de $60,39 \mathrm{~g}$ e o percentual de 10,41\% (Moura et al., 2012). Os resultados elevados podem ser atribuídos a possíveis falhas na quantidade produzida, falta de planejamento correto do número das refeições, superdimensionamento per capita, forma de apresentação das preparações nos balcões de distribuição, preparações incompatíveis com o padrão do cliente ou com seus hábitos alimentares (De Moura et al., 2009; Vaz, 2006)

Tabela 3 - Média de sobra limpa, sobra limpa por pessoa e número de pessoas que poderiam ser alimentadas de uma UAN Passos-MG, 2018.

\begin{tabular}{ccccc}
\hline Dias & Número de Comensais & \% de sobra & Peso da sobra por & PPAA \\
& & & cliente $(\mathrm{g})$ & \\
22 & $133,7 \pm 23,3$ & $32,52 \pm 19,16$ & $166,82 \pm 86,34$ & $41,30 \pm 24,43$ \\
\hline
\end{tabular}

Resultados apresentados em média e desvio padrão. PPAA pessoas poderiam ser alimentadas com o resto acumulado. Fonte: Autores.

Em um estudo realizado em uma UAN na cidade de Nossa Senhora do Socorro-SE, encontraram índice de restoingesta com média de $9,29 \%$ e sobra limpa $3,04 \%$, sendo os restos alimentares deixados pelos comensais suficientes para alimentar 251 pessoas (Barbara et al., 2019).

As UANs devem monitorar as sobras ao longo do tempo, estabelecendo assim um parâmetro ou indicador próprio de segurança, baseando-se em valores apurados nos próprios estabelecimentos, por isso não existe um percentual ideal para sobras em UAN's. Os autores consideram que o excesso das sobras de alimento pode ser influenciado pela oscilação da frequência diária dos comensais, preferências alimentares, treinamento dos funcionários na produção e no porcionamento das refeições (Abreu et al., 2012).

742 pessoas responderam à pesquisa de satisfação que classificou o ambiente, sabor, aparência, temperatura, e variedades das preparações servidas. A ficha de satisfação também apresentava uma questão aberta, para justificar um possível desperdício de refeição servida, para posteriormente correlacionar com os resultados obtidos. Através do tabelamento das respostas, os resultados foram distribuídos conforme as figuras a seguir de acordo com quesito e horário.

Na Figura 2 foram analisados os resultados da pesquisa de satisfação respondida pelos comensais sobre o ambiente do restaurante, referente aos três horários avaliados, tendo 548 votos para ótimo, 184 para bom, 9 para regular e apenas 1 voto para ruim, mostrando sendo considerados satisfatórios (ótimo ou bom) 98,6\%. 
Figura 2: Avaliação do ambiente do restaurante comunitário de uma UAN Passos-MG, 2018.

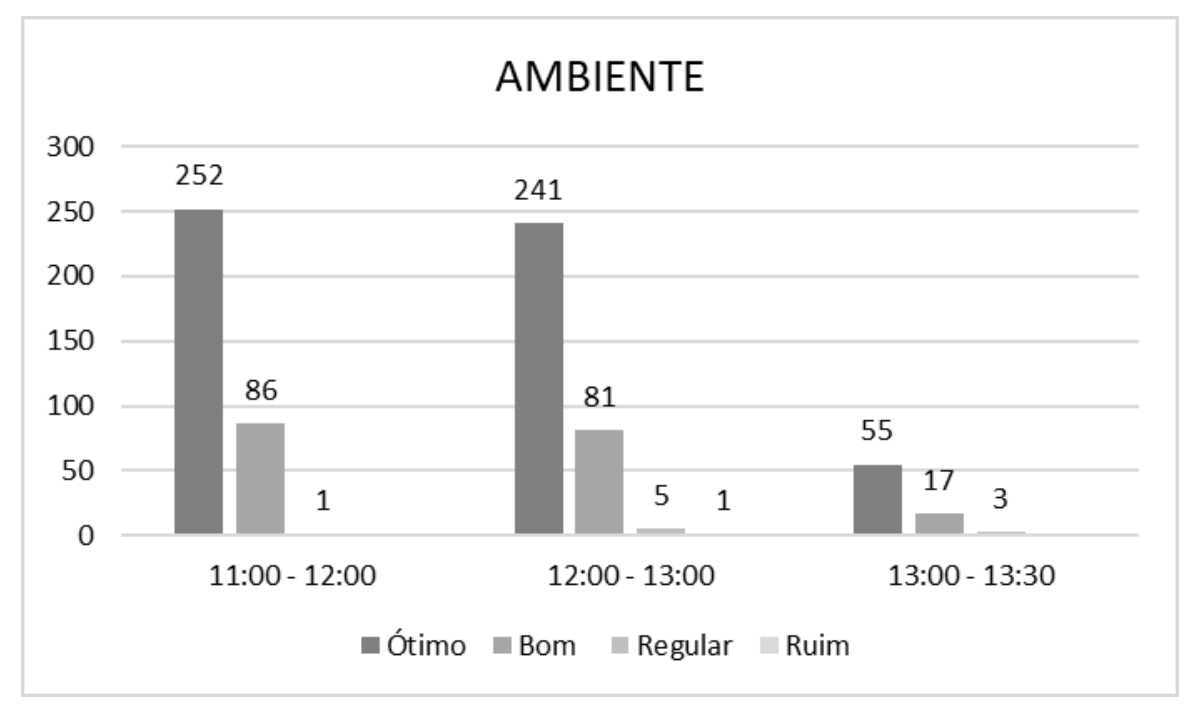

Fonte: Autores.

Em um estudo que avaliou a satisfação de usuários através de uma nota global, observando que o grau de desempenho acompanha o grau de expectativa dos consumidores, não havendo diferença entre as médias para expectativa, 4,65 $( \pm 1,67)$ e satisfação, 4,43 $( \pm 1,57)$ indicando que a maioria dos usuários se encontravam satisfeitos, similarmente ao encontrado no presente estudo (Araújo et al., 2019).

Na Figura 3, foram contabilizados os resultados referentes ao sabor das refeições, sendo considerados satisfatórios (ótimo ou bom) por 95,6\% dos comensais.

Esses resultados são de interesse dos funcionários e colaboradores da UAN estudada, pois, além de conhecerem o paladar dos comensais, são eles que preparam todas as refeições.

Figura 3: Avaliação do Sabor das refeições servidas no restaurante comunitário de uma UAN Passos-MG, 2018.

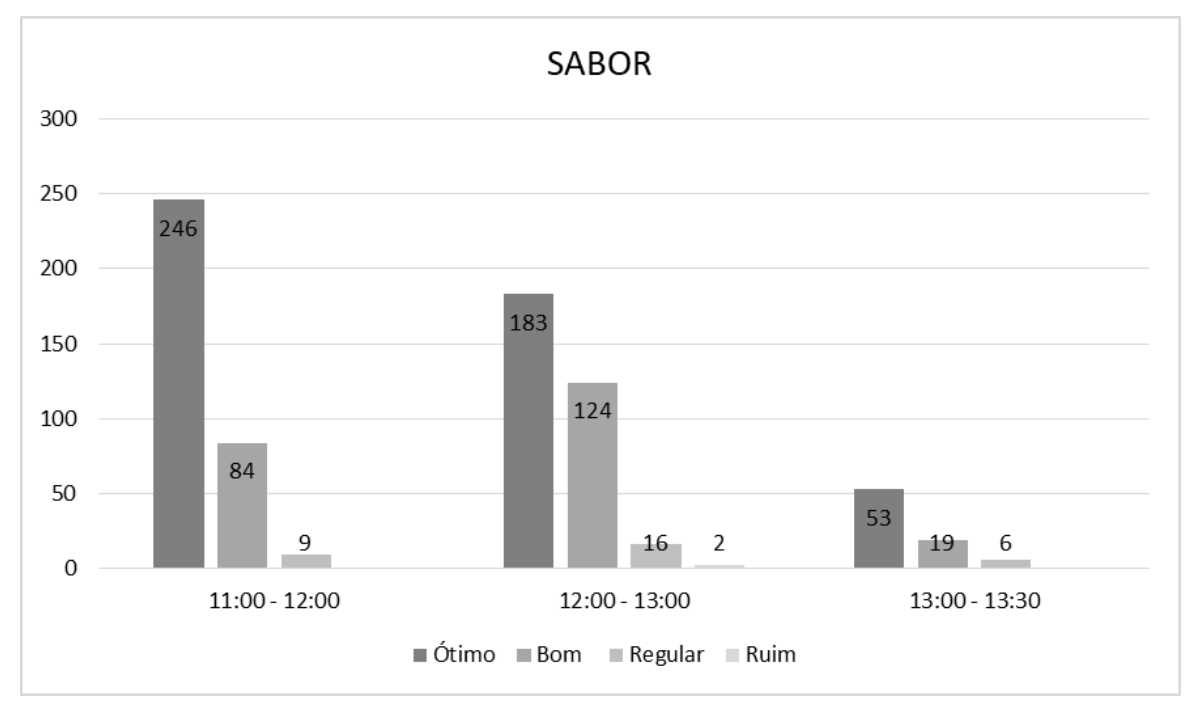

Fonte: Autores (2018).

Ao avaliarem o grau de satisfação dos comensais em relação as refeições oferecidas no restaurante universitário da universidade federal de Santa Maria/RS, por meio de um questionário quantitativo, encontraram um elevado índice de insatisfação com relação ao sabor e tempero, com valores próximos de 45\% (Junior et al., 2015). 
Já em relação à aparência da refeição (Figura 4), 489 dos comensais correspondendo a 65\% responderam que estava ótima, 203 comensais correspondendo a 27,35\% responderam que estava boa, 6,06\% correspondendo a 45 pessoas, disseram que a aparência da refeição era regular, e $0,64 \%$ total de 5 comensais responderam que a aparência era ruim.

Figura 4: Avaliação da Aparência das refeições servidas no restaurante comunitário de uma UAN Passos-MG, 2018.

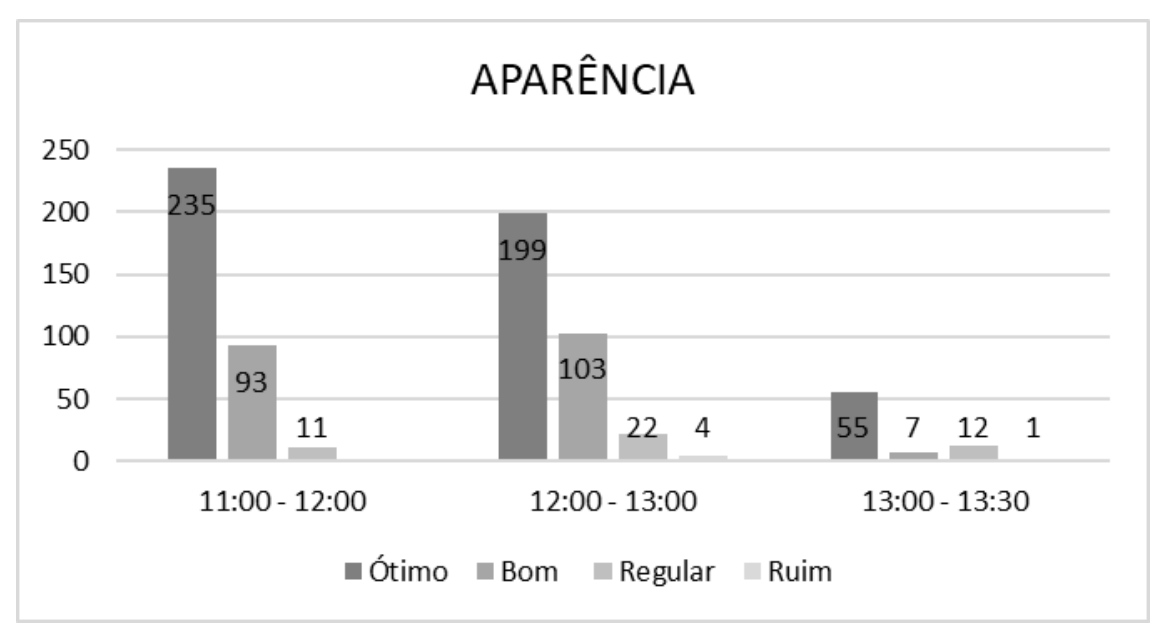

Fonte: Autores (2018).

A Figura 5 apresenta os resultados referentes à temperatura das refeições servidas, neste quesito apesar de 370 votos contabilizados serem ótimos, 242 bons, houve 115 votos para regular e 15 para ruim 15,5\%. Através da ficha de satisfação, também foram observadas reclamações quanto à temperatura e grau de cocção das refeições. A apesar do percentual de aprovação da temperatura ser alto $(82,5 \%)$, é importante investigar as queixas para sanar possíveis problemas e impedir que estes aumentem progressivamente, contribuindo para o desperdício e consequentemente um aumento do resto-ingestão.

Figura 5: Avaliação da Temperatura das refeições servidas no restaurante comunitário de uma UAN Passos-MG, 2018.

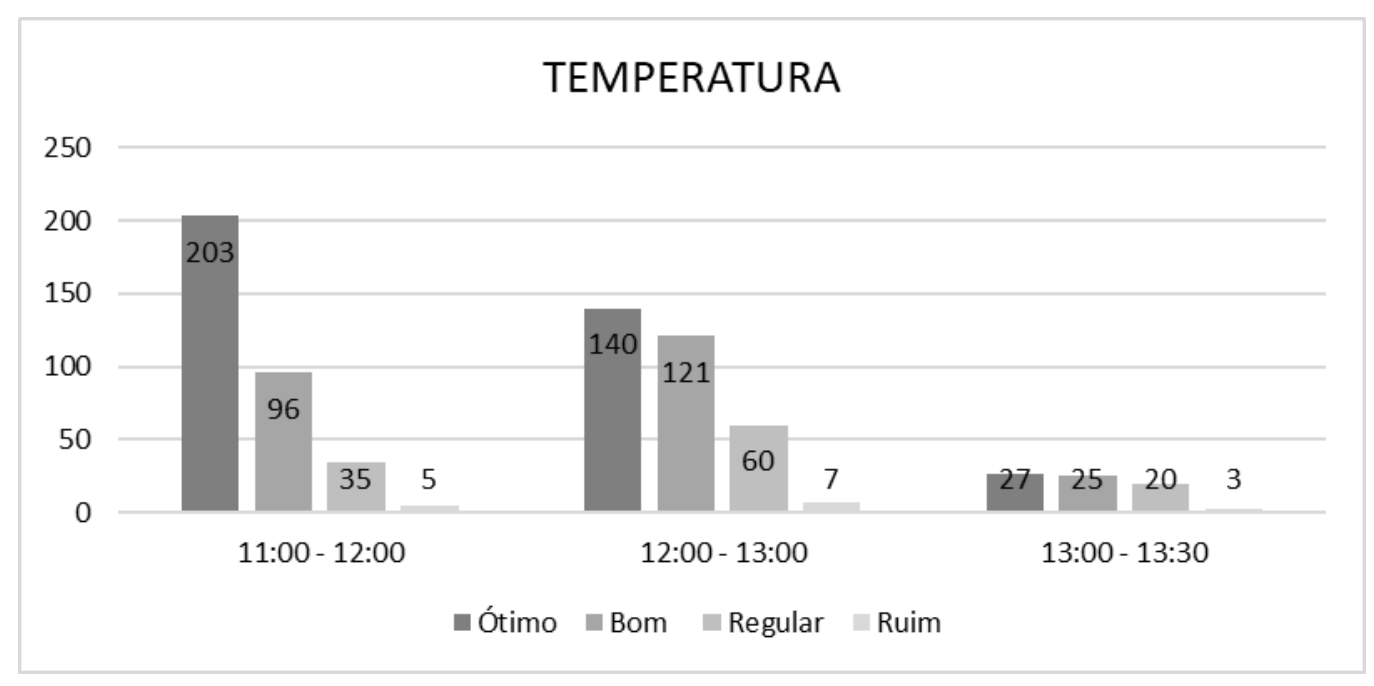

Fonte: Autores (2018).

A Figura 6 apresenta a variedade das preparações, observou-se que a maioria dos votos próximos ao ótimo com 451 votos $60,8 \%$, em seguida foram pontuados 213 votos para bom 28,7\%, totalizando percentuais de satisfação de (ótimo ou bom) com 89,5\%, 63 para regular e 15 para ruim. Estes valores refletem os comentários encontrados na ficha de satisfação onde os comensais elogiaram a qualidade das refeições, relataram boas preparações e a presença de sobremesas. 
Figura 6: Avaliação da Variedade das preparações servidas no restaurante comunitário de uma UAN Passos-MG, 2018.

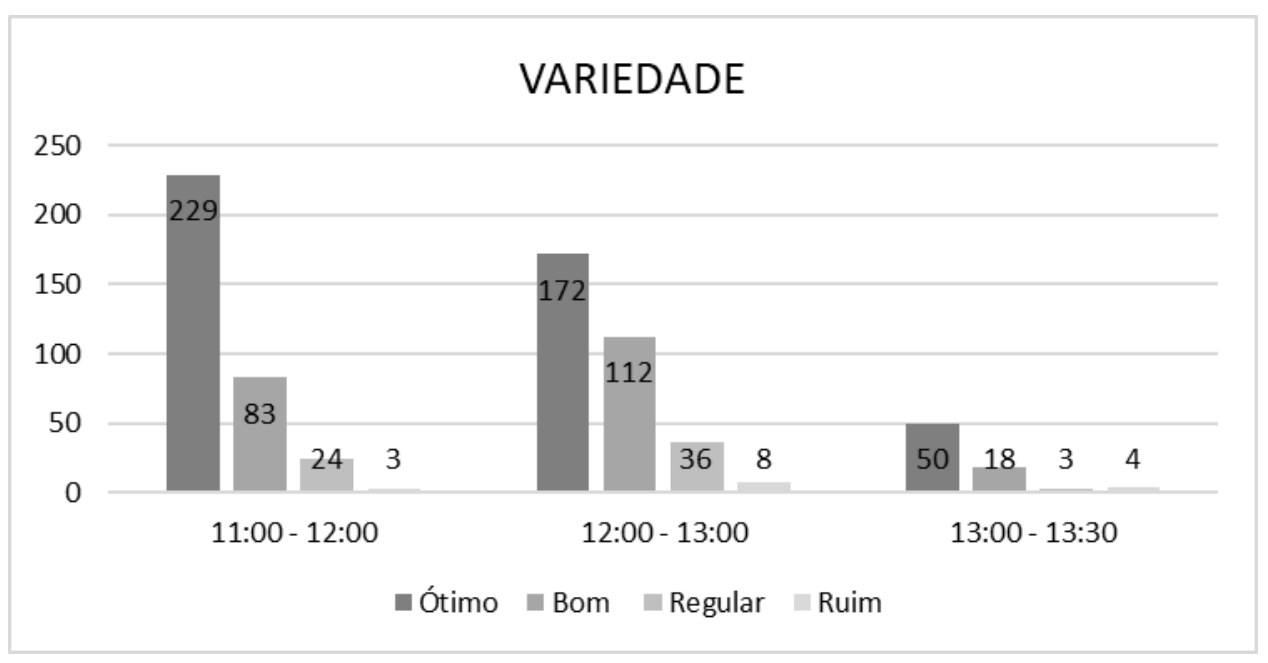

Fonte: Autores (2018).

Os principais atributos ligados à qualidade dos alimentos envolvem a textura dos pratos, apresentação, sabor, qualidade dos ingredientes, aroma, a temperatura dos alimentos, frescor além da higiene (Longart et al., 2018). Em restaurantes de serviço rápido a temperatura e o sabor têm maior relevância na satisfação dos clientes, exigindo monitoramento cuidadoso dessas variáveis (Mathe-Soulek et al., 2015).

Ao ser avaliado a temperatura, aparência e variedade dos alimentos em uma Unidade de alimentação que produz 1600 refeições/dia. Mais de 70\% das respostas dos comensais classificaram estes quesitos como regular ou bom, mostrando maior nível de insatisfação com o serviço prestado quando comparado a esta pesquisa (Cavalcante et al., 2017).

Após análise dos dados, os resultados foram apresentados e discutidos com a nutricionista responsável técnica pela UAN. Diante dos índices satisfatórios de resto ingesta, não houve necessidade de intervenção imediata para sensibilizar os comensais em relação à redução do desperdício. No entanto, devido aos elevados valores de sobra limpa, os dados foram repassados para equipe a fim de estabelecer medidas corretivas que visem reduzir as sobras, como a adequação dos cardápios, das preparações e quantidades produzidas das refeições, treinamento da equipe e conhecimento de hábitos alimentares e culturais dos comensais. Estes aspectos demonstram a necessidade do controle no processo produtivo com a elaboração de rotinas, controle das características sensoriais e monitoramentos das atividades.

O sistema de controle de desperdício permite a descoberta de condutas que geram aumento dos gastos, criando meios e técnicas que planejam o cumprimento de objetivos estipulados de acordo com a necessidade de cada UAN. Sendo assim os registros presentes neste estudo contribuem para implantação de medidas corretivas, podendo servir como subsídio para que se reavaliem o planejamento da produção, aplicando meios e técnicas para redução de custos, lixo desnecessário e o desperdício de alimentos.

\section{Conclusão}

A UAN apresenta elevado índice de desperdício, gerado pelo alto percentual de sobras limpas. Entretanto, o percentual de resto ingesta dos comensais apresenta valores satisfatórios. Dessa forma, faz-se necessário investir em capacitação dos colaboradores, adequação do cardápio, quantidades de produção, entre outras medidas que visem reduzir o desperdício de alimentos e assim minimizar os impactos sociais, econômicos e ambientais. Mais trabalhos que utilizem avaliação do índice de resto-ingesta, sobras limpas e sobra descartada de refeições em Unidades de Alimentação e Nutrição 
bem como treinamentos e campanhas de sensibilização para o controle do desperdício devem ser realizados para auxiliarem em medidas de redução de desperdício e otimização da produtividade.

\section{Referências}

Abreu, E. d., Simony, R., Dias, D., \& Ribeiro. (2012). Avaliação do desperdício alimentar na produção e distribuição de refeições de um hospital de São Paulo. Revista Simbio-Logias, 5(7), 42-50.

Araújo, R. S., Neumann, D., da Rocha Neves, K., \& Soares, H. C. (2019). Satisfação dos clientes do restaurante universitário do campus JK da Universidade federal dos Vales do Jequitinhonha e Mucuri-UFVJM-MG. Brazilian Journal of Development, 5(12), 29971-29989.

Barbara, D. S., dos Santos, G. R., Santana, N. N., da Silva, L. B., Torres Boudou, F. S., \& Filha, E. S. (2019). Avaliação de Sobras Limpas e Resto-Ingesta em uma Unidade de Alimentação e Nutrição na cidade de Nossa Senhora do Socorro-SE. Revista Ciência (In) Cena, 2(9), 52-59.

Borges, M. P., Souza, L. H. R., Pinho, S. d., \& Pinho, L. d. (2019). Impacto de uma campanha para redução de desperdício de alimentos em um restaurante universitário. Engenharia Sanitaria e Ambiental, 24, 843-848.

Brasil, B. J. D. O. R. F. d. (2004). Dispõe sobre Regulamento Técnico de Boas Práticas para Serviços de Alimentação (Resolução RDC nº 216 , de 15 de setembro de 2004). Hig. aliment.

BRASIL, C. F. d. N. (2018). Resolução CFN Nº 600.

Cavalcante, J. M., da Silva, k. L., \& Baratto, I. (2017). Pesquisa de satisfação em um restaurante universitário no Sudoeste do Paranã-PR. RBONE-Revista brasileira de obesidade, nutrição e emagrecimento, 11(68), 661-666.

De Brito, A. M. S., Viera, A. M., Souza, A. B. R., Lopes, A. S., Craveiro, B. A. B., Vasconcelos, F. B., \& Magalhães, M. S. (2016). Avaliação do resto-ingesta de alimentos em uma unidade de alimentação e nutrição no município de Sobral, Ceará. Nutrivisa, 3.

De Moura, P. N., Honaiser, A., \& Bolognini, M. C. M. (2009). Avaliação do índice de Resto-ingesta e sobras em Unidade de Alimentação e Nutrição (UAN) do Colégio Agrícola de Guarapuava/PR. Revista Salus, 3(1), 71-77.

Food, \& Nations, A. O. o. t. U. (2015). Food wastage footprint \& climate change. In.

Galian, L., dos Santos, S. S., \& Madrona, G. S. (2016). Análise do desperdício de alimentos em uma unidade de alimentação e nutrição. REVISTA GEINTECGESTAO INOVACAO E TECNOLOGIAS, 6(2), 3121-3127.

Henz, G. P., \& Porpino, G. (2017). Perdas e desperdício de alimentos: como o Brasil tem enfrentado este desafio global? Horticultura Brasileira, 35(4), 472482 .

Junior, F. d. J. M., Pafiadache, C., Loose, L. H., Piaia, R., Scher, V. T., Peripolli, A., \& Palm, B. (2015). Satisfação dos usuários do restaurante universitário da universidade federal de santa maria: uma análise descritiva. Revista Sociais e Humanas, 28(2), 83-108.

Longart, P., Wickens, E., \& Bakir, A. (2018). An investigation into restaurant attributes: A basis for a typology. International Journal of HospitalityTourism Administration19(1), 95-123.

Machado, C. C. B., Mendes, C. K., de Souza, P. G., Martins, K. d. S. R., \& da Silva, K. C. C. (2012). Avaliação do índice de resto ingesta de uma unidade de alimentação e nutrição institucional de Anápolis-GO. Ensaios e Ciência: ciências biológicas, agrárias e da saúde, 16(6), 151-162.

Mathe-Soulek, K., Slevitch, L., \& Dallinger, I. (2015). Applying mixed methods to identify what drives quick service restaurant's customer satisfaction at the unit-level. International Journal of Hospitality Management, 50, 46-54.

Moura, R. L., Rodrigues, S. d. S., Araújo, E. M. S., do Nascimento, F. R. L., Rodrigues Neto, J. B., \& Stopelle, K. R. d. S. (2012). Avaliação do índice de resto ingestão e sobras em unidade de alimentação e nutrição localizada no campus picos do Instituto federal do Piauí. VII CONNEPI-Congresso Norte Nordeste de Pesquisa e Inovação,

Paiva, D. C. S. D., Nascimento, J. C. D., Cabral, B. E. M., Félix, A. C. F., Lopes, S., \& Estevam, E. (2015). Avaliação do índice de resto-ingestão em uma unidade de alimentação e nutrição de um hospital oncológico após alteração no sistema de distribuição e controle de sobras. Revista Científica da Faminas, 45.

Silva, A. M. d., Silva, C. P., \& Pessina, E. L. (2010). Avaliação do índice de resto ingesta após campanha de conscientização dos clientes contra o desperdício de alimentos em um serviço de alimentação hospitalar. Revista Simbio-Logias, 3(4), 43-56.

Torrent, I. F., da SILVA, L. E. A., Silva, C., dos SANTOS, L. C., \& Pereira, S. C. L. (2018). Desperdício de Alimentos no Ambiente Escolar. Revista Espacios, 39(48), 5.

Vaz, C. S. (2006). Restaurantes: controlando custos e aumentando lucros. Metha, 193.

WFP, F. (2013). IFAD: The State of Food Insecurity in the World 2012. Economic growth is necessary but not sufficient to accelerate reduction of hunger and malnutrition. In: Rome, Italy: IFAD.

Zandonadi, H. S., \& Maurício, A. A. (2012). Avaliação do índice de resto-ingesta, de refeições consumidas por trabalhadores da construção civil no município de Cuiabá, MT. Hig. aliment, 64-70. 